\title{
Evaluation of the Therapeutic Index of a Novel Phosphodiesterase 4B- Selective Inhibitor Over Phosphodiesterase 4D in Mice
}

\author{
Osamu Suzuki ${ }^{1,2, \#, *}$, Kiyoshi Mizukami ${ }^{1}$, Maki Etori ${ }^{1}$, Yoshitaka Sogawa ${ }^{1}$, Nana Takagi ${ }^{1}$, \\ Hiroshi Tsuchida ${ }^{1}$, Kiyoshi Morimoto ${ }^{1}$, Taiji Goto ${ }^{3}$, Toshiharu Yoshino ${ }^{3}$, Tsuyoshi Mikkaichi ${ }^{4}$, \\ Kazuki Hirahara ${ }^{1}$, Satoshi Nakamura ${ }^{2}$, and Hiroaki Maeda ${ }^{1}$ \\ ${ }^{1}$ Biological Research Laboratories III, ${ }^{3}$ Lead Discovery \& Optimization Research Laboratories II, \\ ${ }^{4}$ Drug Metabolism \& Pharmacokinetics Research Laboratories, Daiichi Sankyo Co., Ltd., Tokyo 140-8710, Japan \\ ${ }^{2}$ Department of Bioengineering, Tokyo Institute of Technology, Kanagawa 226-8501, Japan
}

Received June 17, 2013; Accepted September 1, 2013

\begin{abstract}
Phosphodiesterase 4 (PDE4) inhibitors have been developed for the treatment of pulmonary inflammatory diseases, but their clinical use was dose-limited by mainly gastric adverse effects. Recent studies suggested PDE4B-selective inhibitors over PDE4D are supposed to display a wider therapeutic index than subtype non-selective PDE4 inhibitors such as roflumilast. Compound A was identified as an orally active PDE4B-selective inhibitor over PDE4D both in humans (80-fold selective) and mice (29-fold selective). In this study, the therapeutic effects of compound A and roflumilast were evaluated on lipopolysaccaride (LPS) injectioninduced plasma TNF- $\alpha$ elevation and on LPS inhalation-induced pulmonary neutrophilia in mice. The inhibitory effect on gastric emptying in mice was evaluated as a gastric adverse effect. The therapeutic index for TNF- $\alpha$ production $\left(\mathrm{TI}^{\mathrm{TNF}}=\mathrm{ID}_{50}\right.$ in gastric emptying $/ \mathrm{ID}_{50}$ in LPS injection-induced plasma TNF- $\alpha$ elevation) of compound A was larger than roflumilast (9.0 and 0.2 , respectively), whereas the therapeutic index for pulmonary neutrophilia $\left(\mathrm{TI}^{\mathrm{Neu}}=\mathrm{ID}_{50}\right.$ in gastric emptying / $\mathrm{ID}_{50}$ in LPS inhalation-induced pulmonary neutrophilia) of compound $\mathrm{A}$ was comparable to roflumilast (1.0 and 0.5 , respectively). In conclusion, the $\mathrm{TI}^{\mathrm{Neu}}$ of compound $\mathrm{A}$ was not superior compared to that of roflumilast in spite of its high selectivity for PDE4B over PDE4D in mice.
\end{abstract}

Keywords: phosphodiesterase 4B (PDE4B), phosphodiesterase 4D (PDE4D), anti-inflammatory effect, gastric adverse effect, therapeutic index

\section{Introduction}

Phosphodiesterase 4 (PDE4) has been proposed as an attractive target in pulmonary inflammatory diseases such as asthma $(1,2)$ and chronic obstructive pulmonary disease (COPD) $(3,4)$. Several PDE4 inhibitors such as cilomilast and roflumilast have been developed, but clinical use of these PDE4 inhibitors were dose-limited by mainly gastric adverse effects including diarrhea, nausea, and emesis (5). In this regard, next generation

"Present address: Cardiovascular-Metabolics Research Laboratories, Daiichi Sankyo Co., Ltd., Tokyo 140-0005, Japan

*Corresponding author. suzuki.osamu.g5@daiichisankyo.co.jp

Published online in J-STAGE on October 22, 2013

doi: $10.1254 /$ jphs.13103FP
PDE4 inhibitors with a considerable therapeutic effect and limited adverse effect are desired for the treatment of COPD. One hypothesis has been proposed that the cause of emetic response induced by PDE4 subtype nonselective inhibitors is attributed to PDE4D inhibition (6) and that PDE4B-selective inhibitors over PDE4D might have an anti-inflammatory effect with ameliorated gastric adverse effects (7). Although several PDE4Bselective inhibitors have been synthesized along the hypothesis $(8-10)$, it has not been clearly demonstrated whether PDE4B-selective inhibitors display a wider therapeutic index, namely a ratio of therapeutic effects to gastric adverse effects, than non-selective PDE4 inhibitors.

In this study, we report a novel potent PDE4B-selective 
inhibitor over PDE4D named compound A. We investigated whether compound A would show superior therapeutic indices to that of roflumilast by primarily focusing on LPS-induced pulmonary neutrophilia and gastric emptying in mice.

\section{Materials and Methods}

\section{Animals}

Male BALB/c mice aged $8-10$ weeks were purchased from Japan SLC, Inc., Hamamatsu. Mice were used after 1 week of acclimatization in a controlled animal area with a temperature of $23^{\circ} \mathrm{C} \pm 2{ }^{\circ} \mathrm{C}$ and a relative humidity of $55 \% \pm 10 \%$ under a 12 -h cycle of light/dark artificial lighting. Laboratory diets and drinking water were given ad libitum throughout the experiments. All experimental procedures were performed in accordance with the guideline of the Institutional Animal Care and Use Committee at Daiichi Sankyo.

\section{Reagents}

Compound A \{2-[4-[[2-(3-fluoro-4-methoxy-phenyl)-7,8dihydro-6H-thiopyrano [3,2- $d]$ pyrimidin-4-yl] amino] phenyl] acetic acid $\}$, roflumilast, and roflumilast $N$-oxide were synthesized at Daiichi-Sankyo Chemical Research Laboratories (Tokyo). LPS (Escherichia coli O55:B5) and cyclic AMP (cAMP) were obtained from Sigma (St. Louis, MO, USA). The cyclic AMP dynamic 2 kit was obtained from Nihon Schering K.K. (Osaka). Terumeal soft ${ }^{\circledR}$ was obtained from Terumo Corporation (Tokyo). AlphaLISA was obtained from PerkinElmer (Waltham, MA, USA). ACK lysing buffer was obtained from Lonza Walkersville, Inc. (Walkersville, MD, USA). RPMI 1640 medium was obtained from Invitrogen, Inc. (Carlsbad, CA, USA). Human PDE4B2 (full length) and PDE4D2 (full length) were purchased from BPS Bioscience, Inc. (San Diego, CA, USA). Mouse PDE4B2 (amino acid residues 152-564) and PDE4D2 (amino acid residues $79-506$ ) were produced by a standard Escherichia coli expression system.

\section{PDE4 enzyme assay}

A PDE4 inhibitor assay was performed using a cAMP dynamic 2 kit according to the manufacturer's instructions. Briefly, serial dilutions of test compounds with DMSO (100-fold of final concentration) were prepared. Compound solutions were diluted 20-fold with cAMP assay buffer (50 mM Tris, $6 \mathrm{mM} \mathrm{MgCl}_{2}, \mathrm{pH} 7.4$ ). Enzyme solution $(10 \mu \mathrm{l}$ of $30 \mathrm{ng} / \mathrm{ml}$ or cAMP assay buffer for the control) and $5 \mu$ l of test compounds (5-fold concentration of final concentration) were added to each well of the 96-well micro assay plate (Thermo Fisher Scientific, Waltham, MA, USA) and pre-incubated at room temperature for $10 \mathrm{~min}$. The enzymatic reactions were started by adding $10 \mu \mathrm{l}$ of cAMP solution (150 nM in cAMP assay buffer) or cAMP assay buffer and incubated at $37^{\circ} \mathrm{C}$ for $30 \mathrm{~min}$. The enzymatic reaction was terminated by placing the assay plate on ice. The concentration of cAMP was quantified by measurement of fluorescence intensity using EnVision (PerkinElmer) according to the manufacturer's instructions. The $50 \%$ inhibitory concentration $\left(\mathrm{IC}_{50}\right)$ values were determined by nonlinear regression analysis and a sigmoidal dose-response (variable slope) equation using GraphPad Prism 4 (GraphPad Software Inc., San Diego, CA, USA).

\section{LPS-stimulated TNF-a production assay by splenocytes}

An LPS-stimulated TNF- $\alpha$ production assay by splenocytes was performed as previously described (11). Mice were sacrificed by cervical dislocation following deep anesthesia with $50 \mathrm{mg} / \mathrm{kg}$ of sodium pentobarbital and spleens were aseptically removed. Single cell suspensions were prepared by gently teasing each spleen against sterile glass slides and then filtering suspensions through a $70-\mu \mathrm{m}$ cell strainer followed by removing the red blood cells using ACK lysing buffer. The cells were washed three times in ice cold Hanks solution and then resuspended in RPMI 1640 complete medium with $10 \%$ fetal bovine serum. The cells were plated in 96-well plates $\left(4 \times 10^{5}\right.$ cells/well $)$ and incubated with or without LPS $(200 \mathrm{ng} / \mathrm{ml})$ and various concentrations of test compounds. The cells were kept for $24 \mathrm{~h}$ at $37^{\circ} \mathrm{C}$ in a humidified incubator, which maintained a constant atmosphere of $5 \% \mathrm{CO}_{2}$. After 24-h incubation, supernatants were removed and kept at $-80^{\circ} \mathrm{C}$ until measurement. More than $90 \%$ of cell viability was confirmed by an MTT assay. TNF- $\alpha$ concentration of the supernatant was quantified by measurement of fluorescence intensity using AlphaLISA according to the manufacturer's instructions. The $\mathrm{IC}_{50}$ values were also determined by using nonlinear regression analysis and a sigmoidal dose-response (variable slope) equation using GraphPad Prism 4.

\section{LPS injection-induced plasma TNF- $\alpha$ elevation in mice}

LPS injection-induced plasma TNF- $\alpha$ elevation in mice was performed as previously described (12). Overnight-fasted mice were randomly divided into the negative control (vehicle-treated and saline-injected group), positive control (vehicle-treated and LPS-injected group), and test compound groups (test compound-treated and LPS-injected group). The mice were orally administered with test compounds or vehicle alone $(0.5 \%$ methylcellulose) at a volume of $10 \mathrm{ml} / \mathrm{kg}$ at $0.5 \mathrm{~h}$ prior to LPS injection. LPS solution $(2 \mu \mathrm{g} / \mathrm{ml})$ dissolved in saline was injected intravenously into mice at a volume of 250 $\mu 1 /$ animal. One hour following LPS injection, the mice 
were sacrificed by cervical dislocation and blood was sampled into collection tubes with EDTA and kept on ice. After centrifugation, plasma samples were collected and stored at $-80^{\circ} \mathrm{C}$ until use. TNF- $\alpha$ concentration of supernatant was quantified by measurement of fluorescence intensity using AlphaLISA according to the manufacturer's instructions. The $50 \%$ inhibitory dose $\left(\mathrm{ID}_{50}\right)$ (dose that showed 50\% inhibition of vehicle-treated and LPS-injected group) values were calculated by linear regression using Excel 2010 (Microsoft Corporation).

\section{LPS inhalation-induced pulmonary neutrophilia in mice}

LPS inhalation-induced pulmonary neutrophilia in mice was performed as previously described (13). Overnight-fasted mice were randomly divided into negative control (vehicle-treated and saline-inhaled group), positive control (vehicle-treated and LPS-inhaled group), and test compound groups (test compound-treated and LPS-inhaled group). The mice were orally administered with test compounds or vehicle alone $(0.5 \%$ methylcellulose) at a volume of $10 \mathrm{ml} / \mathrm{kg}$. Thirty minutes after administration of the test compounds, the mice were exposed to aerosolized saline or LPS $(0.01 \mathrm{mg} / \mathrm{ml})$. Briefly, the mice were placed in a stainless steel wirecage set inside a plastic exposure chamber. LPS solution was aerosolized for $40 \mathrm{~min}$ with a constant-output nebulizer (Omron Corporation, Kyoto) and all of the output was directed into the exposure chamber. Aerosolized saline was alternatively used for the negative control group. Bronchoalveolar lavage fluid (BALF) collection was performed $5 \mathrm{~h}$ after saline or LPS inhalation. The mice were anesthetized with $50 \mathrm{mg} / \mathrm{kg}$ of sodium pentobarbital intraperitoneally. After semi excision of the trachea, a plastic cannula was inserted and airways were washed 3 times with $1.0 \mathrm{ml}$ of saline to obtain $3 \mathrm{ml}$ of BALF in total. Aliquots of each BALF were centrifuged and the supernatant was removed. The cell pellets were re-suspended in $300 \mu \mathrm{l}$ of saline. The number of neutrophils was counted using a hematology analyzer XT-2000 iv (Sysmex Corporation, Kyoto) and the total number of neutrophils in BAL was quantified. $\mathrm{ID}_{50}$ (dose that showed $50 \%$ inhibition of the vehicle-treated and LPSexposed groups) was calculated by linear regression using Excel 2010.

\section{Gastric emptying in mice}

Evaluation of gastric emptying was performed using the modified method as previously described (14). Overnight-fasted mice were randomly divided into negative control (vehicle-treated group), positive control $(30 \mathrm{mg} / \mathrm{kg}$ of the roflumilast-treated group), and test compound groups. The mice were orally administered with test compounds or vehicle alone $(0.5 \%$ methyl- cellulose) at a volume of $10 \mathrm{ml} / \mathrm{kg}$. Thirty minutes after administration of test compounds, the mice were orally administered with $200 \mu \mathrm{l}$ of a liquid diet $\left(\right.$ Terumeal $^{\circledR}$ soft; Terumo Corporation). One hour after liquid diet administration, the mice were sacrificed by decapitation. After both the entrance and exit were banded, the stomach was extirpated and weighed. ID $_{50}$ (dose that increased stomach weight up to $50 \%$ of $30 \mathrm{mg} / \mathrm{kg}$ of the roflumilast-treated group) values were calculated by linear regression using Excel 2010.

\section{Calculation of the pharmacodynamics index and thera- peutic index}

The therapeutic indices were calculated as follows: The therapeutic index for $\mathrm{TNF}-\alpha$ production $\left(\mathrm{TI}^{\mathrm{TNF}}\right)=$ $\left(\mathrm{ID}_{50}\right.$ in gastric emptying)/( $\mathrm{ID}_{50}$ in LPS injectioninduced plasma TNF- $\alpha$ elevation).

The therapeutic index for pulmonary neutrophilia $\left(\mathrm{TI}^{\mathrm{Neu}}\right)=\left(\mathrm{ID}_{50}\right.$ in gastric emptying $) /\left(\mathrm{ID}_{50}\right.$ in LPS inhalation-induced pulmonary neutrophilia).

\section{Measurement of tissue concentration of test compounds}

Overnight-fasted mice were orally administered with test compounds. Two hours later, blood samples were collected from the tail vein and plasma samples were prepared by centrifugation. The mice were anesthetized by intraperitoneal administration of sodium pentobarbital at a dose of $50 \mathrm{mg} / \mathrm{kg}$. The chest was opened and the lung was perfused via the right ventricular cavity of the heart with saline after cleavage of the abdominal aorta. The lung was isolated, and then it was washed with ice-cold saline briefly and wiped with paper. The tissue samples were weighted and frozen rapidly with dry ice and kept at $-80^{\circ} \mathrm{C}$ until measurement of the compound's concentrations. To quantitate the analytes concentration in plasma and tissues with liquid chromatographytandem mass spectrometry (LC-MSMS), the plasma and tissue homogenates with 4-fold volume of saline were mixed with 10 -fold or more volume of acetonitrile containing niflumic acid as an internal standard. After centrifugation, the aliquots of the supernatant were chromatographed using ultra-fast liquid chromatography (Shimadzu Corporation, Kyoto) on an Xbridge column (C18, dimensions of $2.1 \mathrm{~mm} \times 30 \mathrm{~mm}, 2.5 \mu \mathrm{m}$ particles size; Waters, Milford, MA, USA). Ammonium formate buffer and acetonitrile were used as the mobile phases and eluted with a gradient of acetonitrile. Detection was performed using a Sciex API4000 mass spectrometer with a Turbo-Ion Spray ion source (Sciex, Concord, Canada) in positive or negative ionization mode following multiple-reaction monitoring. 
Data analysis and statistical procedures

Data were calculated using Excel 2000 and expressed as the mean \pm S.E.M. Statistical analysis was performed by a Dunnett's multiple-comparison test using SAS System Release 8.2 (SAS Institute Inc., Cary, NC, USA). A $P$-value of less than 0.05 was considered to be statistically significant.

\section{Results}

Selectivity of compound A for PDE4B and PDE4D enzymes

The inhibitory activities of compound A (chemical structure is depicted in Fig. 1) on PDE4 enzymes were investigated. Compound $\mathrm{A}$ and roflumilast inhibited enzyme activities of PDE4B and PDE4D in a concentration-dependent manner both in humans and mice (Fig. 2), and the $\mathrm{IC}_{50}$ values are shown in Table 1 . In addition, selectivity of compound A for human and mouse PDE4B were calculated by dividing the $\mathrm{IC}_{50}$ value of PDE4D by the $\mathrm{IC}_{50}$ value of PDE4B and compared with that of roflumilast (Table 1). Roflumilast did not show selectivity either for human or mouse PDE4B (1.0- and 0.61 -fold, respectively) as previously reported (10). In

A

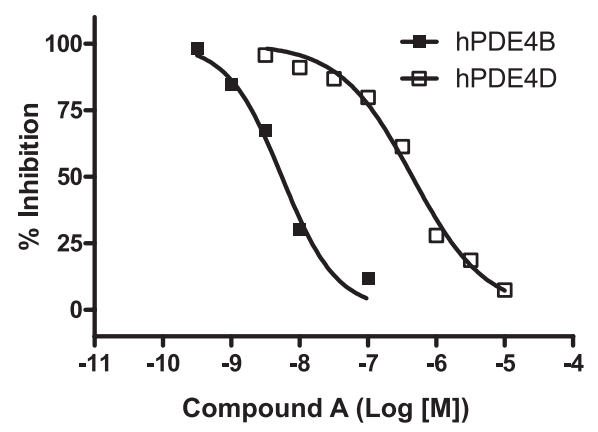

C

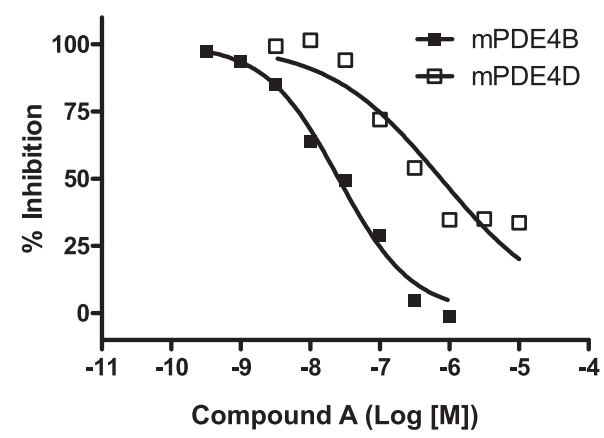

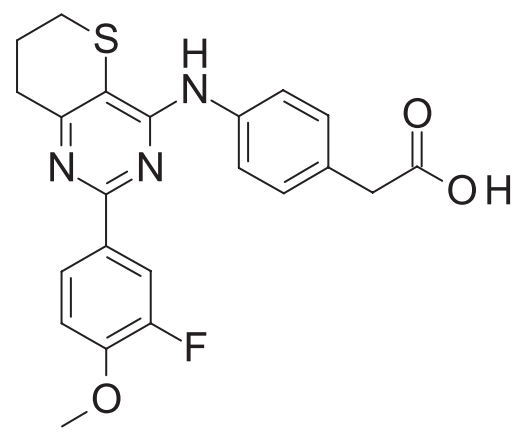

Fig. 1. Chemical structure of compound A (2-[4-[[2-(3-fluoro-4methoxy-phenyl)-7,8-dihydro-6H-thiopyrano[3,2-d]pyrimidin-4-yl] amino]phenyl]acetic acid).

contrast, compound A was highly selective for PDE4B over PDE4D both in humans and mice (80- and 29-fold, respectively).

Inhibitory effect of compound A on LPS-stimulated TNF- $\alpha$ production by mouse splenocytes

The inhibitory effect of compound A on LPS-stimulated TNF- $\alpha$ secretion by mouse splenocytes was tested. Compound A and roflumilast inhibited TNF- $\alpha$ produc-
B

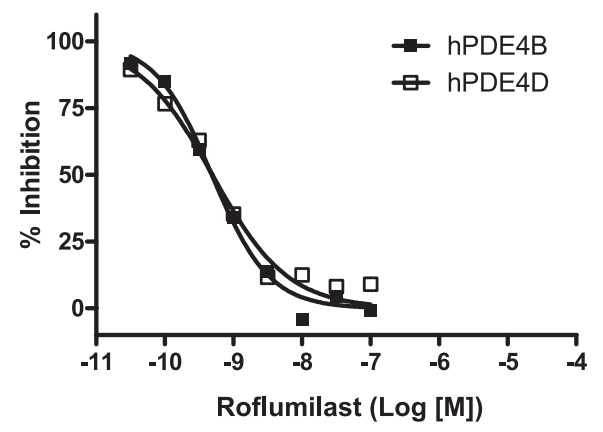

D

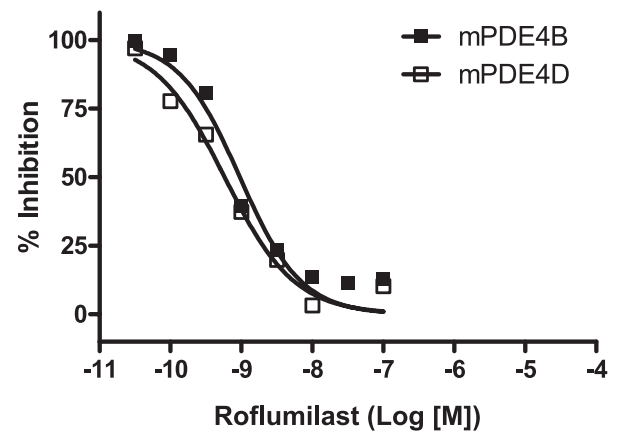

Fig. 2. Inhibitory activities of compound $A$ for PDE4B and PDE4D enzymes. The inhibitory activities of compound A and roflumilast are shown for PDE4B and PDE4D in humans (A, B) and in mice (C, D), respectively. The enzyme activities were measured by monitoring the degradation of $\mathrm{cAMP}$ and the remaining ratio of cAMP with each concentration of the test compounds shown. The figures show one representative of more than two independent experiments. 
Table 1. Summary of pharmacological profile of compound A in vitro

\begin{tabular}{|c|c|c|c|c|c|}
\hline \multirow{2}{*}{ Species } & \multirow{2}{*}{ Compound } & \multicolumn{2}{|c|}{$\mathrm{IC}_{50}(\mathrm{nM})$} & \multirow{2}{*}{$\begin{array}{l}\text { Selectivity } \\
\text { (b)/(a) }\end{array}$} & \multirow{2}{*}{$\begin{array}{c}\mathrm{IC}_{50}(\mathrm{nM}) \\
\mathrm{TNF}-\alpha^{\mathrm{a}}\end{array}$} \\
\hline & & PDE4B (a) & PDE4D (b) & & \\
\hline \multirow[t]{2}{*}{ Human } & Compound A & 5.5 & 440 & 80 & - \\
\hline & Roflumilast & 0.49 & 0.49 & 1.0 & - \\
\hline \multirow[t]{2}{*}{ Mouse } & Compound A & 26 & 760 & 29 & 11 \\
\hline & Roflumilast & 0.95 & 0.58 & 0.61 & 1.2 \\
\hline
\end{tabular}

${ }^{a}$ TNF- $\alpha$ production by mouse splenocytes stimulated with $200 \mathrm{ng} / \mathrm{ml}$ of LPS for $24 \mathrm{~h}$.

tion in a concentration-dependent manner (data not shown) with the $\mathrm{IC}_{50}$ values of 11 and $1.2 \mathrm{nM}$, respectively (Table 1).

Inhibitory effect of compound A on LPS injection-induced plasma TNF- $\alpha$ elevation in mice

The inhibitory effect of compound A on LPS injectioninduced plasma TNF- $\alpha$ elevation in mice was tested. Oral administration of compound $\mathrm{A}$ and roflumilast to mice before LPS injection dose-dependently inhibited elevation of the plasma concentration of TNF- $\alpha$ (Fig. 3). The $\mathrm{ID}_{50}$ values of compound A and roflumilast were $29 \mathrm{mg} / \mathrm{kg}$ and $23 \mathrm{mg} / \mathrm{kg}$, respectively. The potency of compound A and roflumilast were almost identical in this evaluation.

\section{Inhibitory effects of compound A on LPS inhalation- induced pulmonary neutrophilia in mice \\ The inhibitory effect of compound A on LPS inhala-} tion-induced pulmonary neutrophilia was investigated in mice. Oral administration of compound A and roflumilast to mice before LPS inhalation reduced the number of neutrophils in BALF in a dose-dependent manner (Fig. 4). The $\mathrm{ID}_{50}$ values for compound $\mathrm{A}$ and roflumilast on LPS inhalation-induced pulmonary neutrophilia were $253 \mathrm{mg} / \mathrm{kg}$ and $7.2 \mathrm{mg} / \mathrm{kg}$, respectively. When comparing the $\mathrm{ID}_{50}$ values, compound A required an 8.7 times higher dose to demonstrate the inhibitory effect on LPS inhalation-induced pulmonary neutrophilia than on LPS-induced plasma TNF- $\alpha$ elevation, whereas roflumilast showed almost the same or stronger potency in this evaluation. To rule out the possibility that this difference of potency is due to a difference of pharmacokinetic profile or distribution of the compounds, we analyzed the plasma concentration and the lung tissueto-plasma concentration ratio (Kp). The plasma concentration of compound A sustained a sufficient level to inhibit PDE4B over $4 \mathrm{~h}$ after oral administration of compound $\mathrm{A}$ at a dose of $30 \mathrm{mg} / \mathrm{kg}$ (data not shown). In addition, no significant difference was found in lung distribution between compound $\mathrm{A}$, roflumilast, and roflumilast $\mathrm{N}$-oxide, a dominant active metabolite of
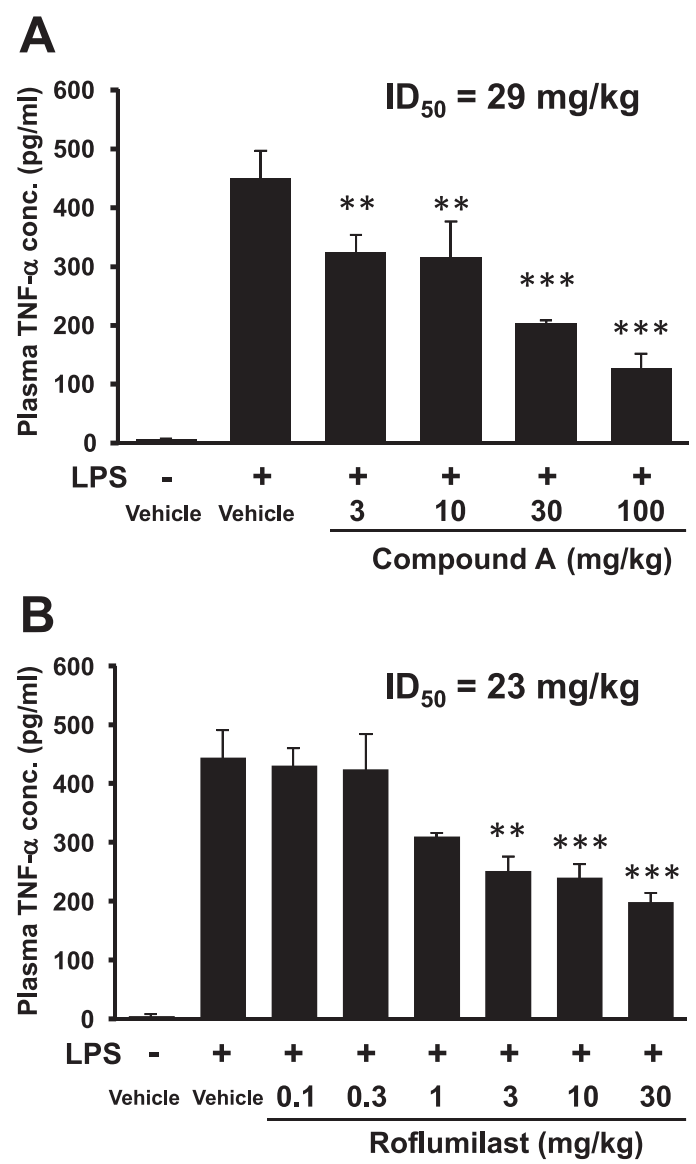

Fig. 3. Inhibitory effect of compound A on LPS injection-induced TNF- $\alpha$ elevation in plasma. Mice were pretreated with test compounds or vehicle alone $30 \mathrm{~min}$ before LPS injection. The plasma samples were collected $1 \mathrm{~h}$ after saline or LPS injection and the concentration of TNF- $\alpha$ was measured. The inhibitory effect of compound A (A) and roflumilast (B) are shown. The figures show one representative of more than two independent experiments. Data are expressed as the mean \pm S.E.M. $(\mathrm{n}=6$ per group, $* * P<0.01$, $* * * P<0.001)$.

roflumilast $(K p$ lung $=0.26 \pm 0.01,0.33 \pm 0.11$, and $0.16 \pm 0.05$, respectively). Therefore, weaker potency of compound A than roflumilast in LPS inhalationinduced pulmonary neutrophilia is not due to faster 

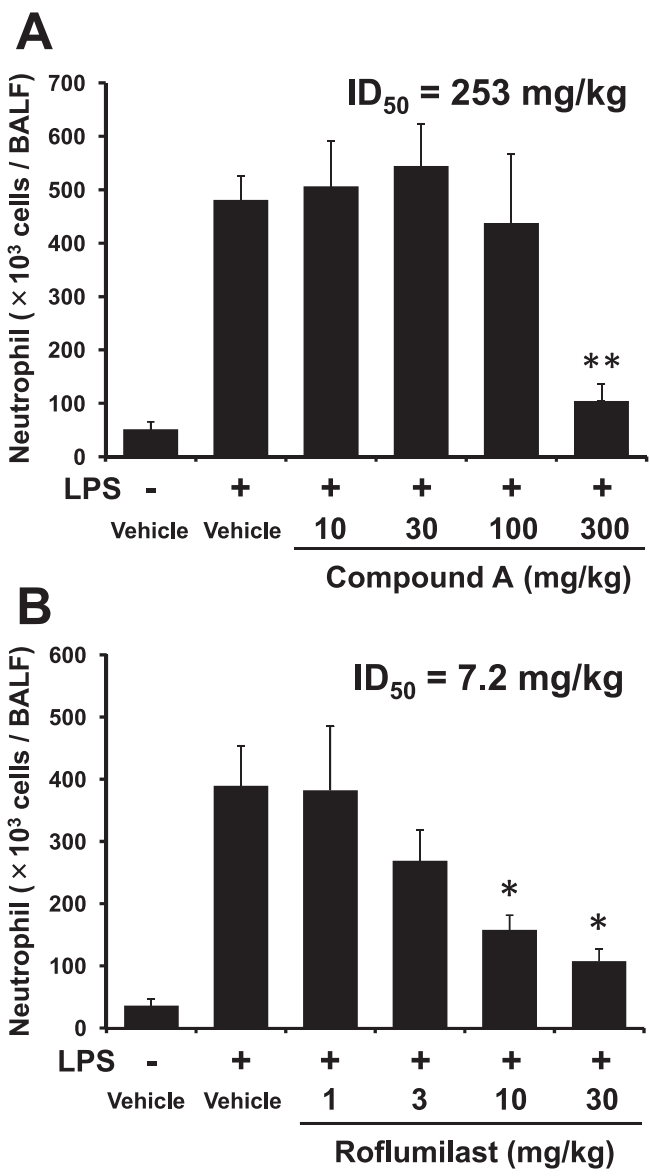

Fig. 4. Inhibitory effect of compound A on LPS inhalation-induced pulmonary neutrophilia. Mice were pretreated with test compounds or vehicle alone $30 \mathrm{~min}$ before the aerosolized LPS challenge. BALF was collected $5 \mathrm{~h}$ after saline or LPS inhalation and the number of neutrophils was counted. The inhibitory effect of compound A (A) and roflumilast (B) are shown. The figures show one representative of more than three independent experiments. Data are expressed as the mean \pm S.E.M. $(\mathrm{n}=7-9$ per group, $* P<0.05, * * P<0.01)$.

clearance or lower exposure of compound A to the lung than roflumilast.

\section{Inhibitory effect of compound A on gastric emptying}

The inhibitory effect of compound A on gastric emptying, which is one of the gastric adverse effects of roflumilast (15), was investigated in mice. Oral administration of compound A or roflumilast to mice before oral administration of a liquid diet increased the stomach weight in a dose-dependent manner, indicating delayed gastric emptying (Fig. 5). The $\mathrm{ID}_{50}$ values for compound $\mathrm{A}$ and roflumilast in gastric emptying were $260 \mathrm{mg} / \mathrm{kg}$ and $3.9 \mathrm{mg} / \mathrm{kg}$, respectively. Compound A required a 67-fold higher dose to induce the delay of gastric emptying than roflumilast.
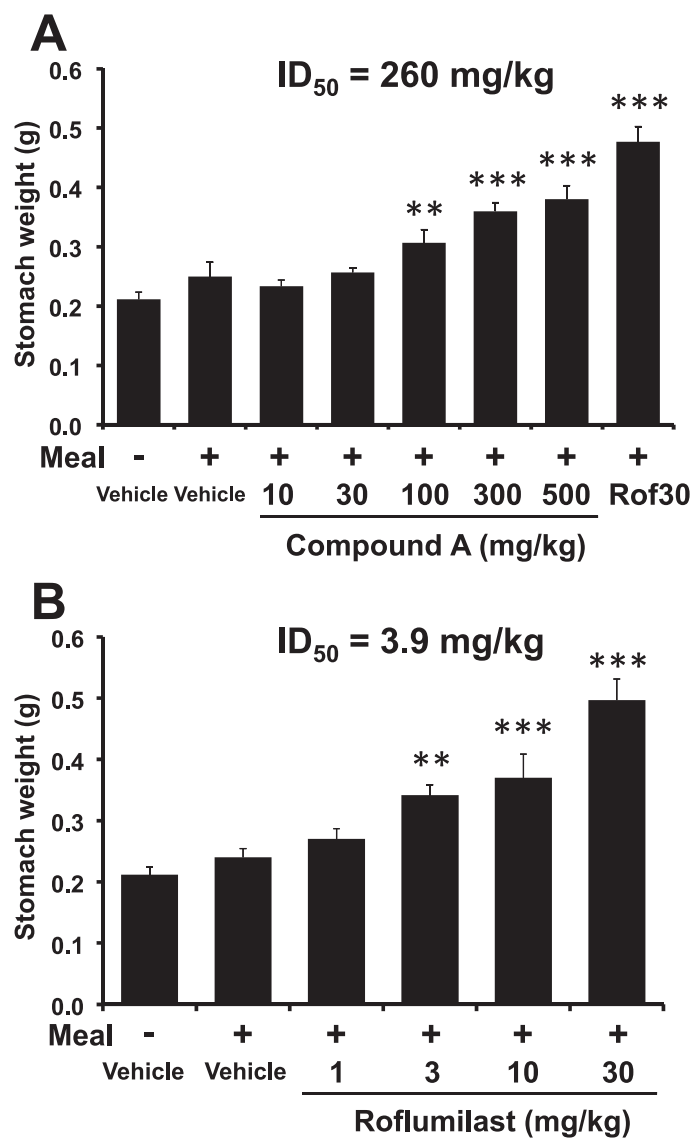

Fig. 5. Inhibitory effect of compound A on gastric emptying. Mice were pretreated with test compounds or vehicle alone. Thirty minutes following test compound pretreatment, mice were orally administered $200 \mu \mathrm{l}$ of a liquid diet and sacrificed $1 \mathrm{~h}$ later and then the stomach was weighed. The inhibitory effect of compound A (A) and roflumilast (B) are shown. "Rof30" indicates a group of mice orally treated with $30 \mathrm{mg} / \mathrm{kg}$ of roflumilast. The figures show one representative of more than three independent experiments. Data are expressed as the mean \pm S.E.M. $(\mathrm{n}=6$ per group, $* * P<0.01, * * * P<0.001)$.

\section{Therapeutic index of compound $A$}

The therapeutic indices for LPS-induced TNF- $\alpha$ production $\left(\mathrm{TI}^{\mathrm{TNF}}\right)$ and pulmonary neutrophilia $\left(\mathrm{TI}^{\mathrm{Neu}}\right)$ were calculated by dividing the $\mathrm{ID}_{50}$ value in gastric emptying by the $\mathrm{ID}_{50}$ value in LPS injection-induced TNF- $\alpha$ elevation and LPS inhalation-induced neutrophilia, respectively (Table 2). The $\mathrm{TI}^{\mathrm{TNF}}$ of compound A and roflumilast were 9.0 and 0.2 , respectively, indicating that compound A showed a reduced gastric adverse effect. In contrast, the $\mathrm{TI}^{\mathrm{Neu}}$, a more important parameter than $\mathrm{TI}^{\mathrm{TNF}}$ regarding airway inflammation, of compound A and roflumilast were almost identical (1.0 and 0.5 , respectively). This was because compound A showed less efficacy in suppressing pulmonary neutrophilia than roflumilast despite the fact that compound A showed a reduced gastric adverse effect. 
Table 2. Summary of profile of compound A in vivo

\begin{tabular}{llcc}
\hline & & Compound A & Roflumilast \\
\hline $\mathrm{ID}_{50}(\mathrm{mg} / \mathrm{kg})$ & Plasma TNF- $\alpha$ production (a) & 29 & 23 \\
& Pulmonary neutrophilia (b) & 253 & 7.2 \\
& Gastric emptying (c) & 260 & 3.9 \\
& Therapeutic index for TNF- $\alpha$ production $\left(\mathrm{TI}^{\mathrm{TNF}}\right)=(\mathrm{c}) /(\mathrm{a})$ & 9.0 & 0.2 \\
& Therapeutic index for pulmonary neutrophilia $\left(\mathrm{TI}^{\mathrm{Neu}}\right)=(\mathrm{c}) /(\mathrm{b})$ & 1.0 & 0.5 \\
\hline
\end{tabular}

\section{Discussion}

The purpose of this study was to elucidate whether a PDE4B-selective inhibitor over PDE4D displays a wider therapeutic index than roflumilast, a subtype nonselective PDE4 inhibitor, in mouse models for airway inflammation and gastric adverse response. We evaluated the therapeutic indices of compound A, a novel PDE4B inhibitor, by comparing the $\mathrm{ID}_{50}$ values in LPS inhalation-induced neutrophilia with that in gastric emptying. We found that compound A did not possess wider $\mathrm{TI}^{\mathrm{Neu}}$ compared to roflumilast. To our knowledge, this is the first report that showed both the therapeutic effect and gastric adverse effect of a PDE4B-selective inhibitor over PDE4D using animals in which the inhibitory activities of the compound against PDE4B and PDE4D were verified.

In an enzyme assay, compound A showed 29-fold selectivity for mouse PDE4B over mouse PDE4D. In a cell-based assay, the $\mathrm{IC}_{50}$ value of compound $\mathrm{A}$ for TNF- $\alpha$ secretion by mouse splenocytes $(11 \mathrm{nM})$ was almost the same as that for mPDE4B $(26 \mathrm{nM})$ and was far below that for mPDE4D $(760 \mathrm{nM})$, indicating that mPDE4B but not mPDE4D is responsible for LPSstimulated TNF- $\alpha$ production by mouse splenocytes. This observation is consistent with previous reports using PDE4B and PDE4D gene knockout mice that demonstrated that PDE4B but not PDE4D is responsible for LPS-stimulated TNF- $\alpha$ production $(16,17)$.

The inhibitory effects of compound A on LPS inhalation-induced pulmonary neutrophilia and gastric emptying in mice were evaluated to calculate the $\mathrm{TI}^{\mathrm{Neu}}$. We found that the inhibitory potency of compound A on gastric emptying in mice was weaker than that of roflumilast, suggesting that PDE4D could be involved in gastric motility. It was rather surprising that the $\mathrm{TI}^{\mathrm{Neu}}$ of compound A was almost identical to that of roflumilast since the $\mathrm{TI}^{\mathrm{TNF}}$ of compound $\mathrm{A}$ was improved more than that of roflumilast by 45 -fold. The difference between the two indices is attributed to weak potency of compound A on LPS inhalation-induced pulmonary neutrophilia compared to LPS injection-induced plasma
TNF- $\alpha$ elevation. This weak potency of compound A in inhibiting LPS inhalation-induced neutrophilia was neither due to a short half-life in plasma concentration (data not shown) nor lower distribution to the lung than roflumilast. A plausible explanation why compound A did not effectively inhibit neutrophil mobilization would be the existence of other mediators other than TNF- $\alpha$ or the complexity of the inflammatory process of neutrophil infiltration to the lung in which not only PDE4B but PDE4D would be involved. This observation is consistent with a previous study that evaluated PDE4Bgene- and PDE4D-gene-deficient mice in which LPSinduced neutrophil accumulation in BALF was similarly decreased in PDE4B- or PDE4D-deficient mice; thus both PDE4B and PDE4D inhibition are required to fully suppress neutrophil infiltration (18). Taken together, compound A inhibited TNF- $\alpha$ production by pro-inflammatory cells such as monocytes or macrophages, which predominantly depends on PDE4B but could not inhibit neutrophil trafficking to the inflammatory sites effectively which depends on both PDE4B and PDE4D.

Our observation is not consistent with the previous study by other investigators in which they demonstrated that human PDE4B-selective inhibitors over PDE4D showed a wider therapeutic index than cilomilast in ferrets (9). We speculate that mechanisms of neutrophil activation and trafficking to the lung might be different between mice and ferrets, and PDE4D might not play a major role in neutrophil accumulation to the lung in ferrets. If there are differences in mechanisms of neutrophil activation among species, it is important to clarify which subtype of PDE4 enzymes plays a major role in neutrophil activation in humans. Further detailed studies using human inflammatory cells are required to determine the therapeutic potential of PDE4B-selective inhibitors for the treatment of COPD.

Neutrophilic inflammation in the lung has been considered to be one of the critical factors in disease progression of COPD, and neutrophil counts in induced sputum have been used as a biomarker $(19,20)$. Although evidence has recently been provided that the abnormalities seen in COPD are not only restricted to the airways 
but are also systemically present (21), the impact of systemic elevation of inflammatory mediators such as TNF- $\alpha$, IL- 8 , or IL- 6 on disease progression in COPD is still unclear. Clinical studies indicating that neither anti-TNF- $\alpha$ antibody infliximab (22) nor anti-IL-8 antibody (23) delayed the decline of the lung function in patients with COPD implies that at least suppression of one inflammatory mediator may not be enough to delay disease progression of COPD. Considering these results, compound A which possesses weak potency to neutrophilic inflammation might not be sufficient to inhibit decline of the lung function in patients with COPD even if it attenuated systemic TNF- $\alpha$ production.

In conclusion, we identified a novel potent PDE4Bselective inhibitor both in humans and mice. However, the therapeutic index of compound A calculated by the inhibitory effect on LPS inhalation-induced neutrophilia and gastric emptying was not superior compared to that of roflumilast in mice.

\section{Acknowledgments}

We are grateful to Dr. Tomomi Yoshitomi, Ms. Tomoko Terauchi, and Mr. Kenichi Obata for their contributions to this work.

\section{References}

1 Torphy TJ. Phosphodiesterase isozymes: molecular targets for novel antiasthma agents. Am J Respir Crit Care Med. 1998; 157:351-370.

2 Page CP, Spina D. Phosphodiesterase inhibitors in the treatment of inflammatory diseases. Handb Exp Pharmacol. 2011;204: 391-414.

3 Diamant Z, Spina D. PDE4-inhibitors: a novel, targeted therapy for obstructive airways disease. Pulm Pharmacol Ther. 2011; 24:353-360.

4 Hatzelmann A, Morcillo EJ, Lungarella G, Adnot S, Sanjar S, Beume R, et al. The preclinical pharmacology of roflumilast--a selective, oral phosphodiesterase 4 inhibitor in development for chronic obstructive pulmonary disease. Pulm Pharmacol Ther. 2010;23:235-256.

5 Lipworth BJ. Phosphodiesterase-4 inhibitors for asthma and chronic obstructive pulmonary disease. Lancet. 2005;365:167175.

6 Robichaud A, Stamatiou PB, Jin SL, Lachance N, MacDonald D, Laliberté F, et al. Deletion of phosphodiesterase 4D in mice shortens alpha(2)-adrenoceptor-mediated anesthesia, a behavioral correlate of emesis. J Clin Invest. 2002;110:10451052 .

7 Jin SL, Ding SL, Lin SC. Phosphodiesterase 4 and its inhibitors in inflammatory diseases. Chang Gung Med J. 2012;35:197210 .

8 Kranz M, Wall M, Evans B, Miah A, Ballantine S, Delves C, et al. Identification of PDE4B over 4D subtype-selective inhibitors revealing an unprecedented binding mode. Bioorg Med Chem. 2009;17:5336-5341.
9 Naganuma K, Omura A, Maekawara N, Saitoh M, Ohkawa N, Kubota T, et al. Discovery of selective PDE4B inhibitors. Bioorg Med Chem Lett. 2009;19:3174-3176.

10 Claveau D, Chen SL, O'Keefe S, Zaller DM, Styhler A, Liu S, et al. Preferential inhibition of $\mathrm{T}$ helper 1 , but not $\mathrm{T}$ helper 2 , cytokines in vitro by L-826,141 [4-[2-(3,4-Bisdifluromethoxyphenyl)-2-[4-(1,1,1,3,3,3-hexafluoro-2-hydroxypropan-2-yl)phenyl]-ethyl]3-methylpyridine-1-oxide], a potent and selective phosphodiesterase 4 inhibitor. J Pharmacol Exp Ther. 2004; 310:752-760.

11 Petursdottir DH, Hardardottir I. Dietary fish oil increases the number of splenic macrophages secreting TNF-alpha and IL-10 but decreases the secretion of these cytokines by splenic T cells from mice. J Nutr. 2007;137:665-670.

12 Ma Z, Li J, Yang L, Mu Y, Xie W, Pitt B, et al. Inhibition of LPS- and CpG DNA-induced TNF-alpha response by oxidized phospholipids. Am J Physiol Lung Cell Mol Physiol. 2004; 286:L808-L816.

13 Basit A, Reutershan J, Morris MA, Solga M, Rose CE Jr, Ley K. ICAM-1 and LFA-1 play critical roles in LPS-induced neutrophil recruitment into the alveolar space. Am J Physiol Lung Cell Mol Physiol. 2006 ;291:L200-L207.

14 Mine Y, Oku S, Yoshida N. Anti-emetic effect of mosapride citrate hydrate, a 5-HT4 receptor agonist, on selective serotonin reuptake inhibitors (SSRIs)-induced emesis in experimental animals. J Pharmacol Sci. 2013;121:58-66.

15 Product Monograph ${ }^{\text {Pr}} \mathrm{DAXAS}{ }^{\mathrm{TM}}$ (http://www.takedacanada. $\mathrm{com} / \mathrm{ca} / \sim / \mathrm{media} /$ Countries/ca/Files/Product\%20PDFs/ Daxas\%20English\%20Distribution\%2031-May\%202011with\%20logo.pdf)

16 Jin SL, Conti M. Induction of the cyclic nucleotide phosphodiesterase PDE4B is essential for LPS-activated TNF-alpha responses. Proc Natl Acad Sci U S A. 2002;28:7628-7633.

17 Jin SL, Lan L, Zoudilova M, Conti M. Specific role of phosphodiesterase 4B in lipopolysaccharide-induced signaling in mouse macrophages. J Immunol. 2005;175:1523-1531.

18 Ariga M, Neitzert B, Nakae S, Mottin G, Bertrand C, Pruniaux $\mathrm{M}$, et al. Nonredundant function of phosphodiesterase 4D and 4B in neutrophil recruitment to the site of inflammation. J Immunol. 2004;173:7531-7538.

19 O'Donnell RA, Peebles C, Ward JA, Daraker A, Angco G, Broberg $\mathrm{P}$, et al. Relationship between peripheral airway dysfunction, airway obstruction, and neutrophilic inflammation in COPD. Thorax. 2004;59:837-842.

20 Cowburn AS, Condliffe AM, Farahi N, Summers C, Chilvers ER. Advances in neutrophil biology: clinical implications. Chest. 2008; 134:606-612.

21 Sinden NJ, Stockley RA. Systemic inflammation and comorbidity in COPD: a result of 'overspill' of inflammatory mediators from the lungs? Review of the evidence. Thorax. 2010;65: 930-936.

22 Rennard SI, Fogarty C, Kelsen S, Long W, Ramsdell J, Allison J, et al. The safety and efficacy of infliximab in moderate to severe chronic obstructive pulmonary disease. Am J Respir Crit Care Med. 2007;175:926-934.

23 Mahler DA, Huang S, Tabrizi M, Bell GM. Efficacy and safety of a monoclonal antibody recognizing interleukin-8 in COPD: a pilot study. Chest. 2004;126:926-934. 